\title{
TOPOLOGY ON THE SPACES OF ORDERINGS OF GROUPS
}

\author{
ADAM S. SIKORA
}

\begin{abstract}
A natural topology on the space of left orderings of an arbitrary semi-group is introduced. It is proved that this space is compact and that for free abelian groups it is homeomorphic to the Cantor set. An application of this result is a new proof of the existence of universal Gröbner bases.
\end{abstract}

\section{ORderings FOR SEMI-GROUPS}

Given a semi-group $G$ (ie. a set with an associative binary operation), a linear order, $<$, on $G$ is a left order if $a<b$ implies $c a<c b$, for any $c$. Similarly, a linear order , $<$, is a right order if $a<b$ implies $a c<b c$, for any $c \in G$. The sets of all left and right orderings of $G$ are denoted by $L O(G)$ and $R O(G)$ respectively. If $G$ is a group then there is a 1-1 correspondence between these two sets which associates with any left ordering, $<_{l}$, a right ordering, $<_{r}$, such that $a<_{r} b$ if and only if $b^{-1}<_{l} a^{-1}$. For more about ordering of groups see [4, 7, 8].

Let $U_{a, b} \subset L O(G)$ denote the set of all left orderings, $<$, for which $a<b$. We can put a topology on $L O(G)$ in one of the following two ways.

Definition 1.1. $L O(G)$ has the smallest topology for which all the sets $U_{a, b}$ are open. Any open set in this topology is a union of sets of the form $U_{a_{1}, b_{1}} \cap \ldots \cap U_{a_{n}, b_{n}}$.

Definition 1.2. Let $G_{0} \subset G_{1} \subset G_{2} \ldots \subset G$ be an arbitrary complete filtration of $G$ by its subsets. (A filtration is complete if $\bigcup_{i} G_{i}=G$ ). For $<_{1},<_{2} \in L O(G)$ we define $\rho\left(<_{1},<_{2}\right)$ to be $\frac{1}{2^{r}}$, where $r$ is the largest number with the property that $<_{1}$ and $<_{2}$ coincide when restricted to $G_{r}$; We put $\rho\left(<_{1},<_{2}\right)=0$ if such $r$ does not exist $(r=\infty)$.

From now on we will consider countable semi-groups $G$ only and such filtrations only which are composed of finite subsets of $G$.

Proposition 1.3. $\rho$ is a metric on $L O(G)$ and the topology on $L O(G)$ induced by that metric coincides with the topology introduced in Definition [1.1. In particular, it does not depend on the choice of a filtration of $G$.

1991 Mathematics Subject Classification. Primary: 06F15, 13P10, Secondary: 06F05, $20 \mathrm{~F} 60$. 
Proof. It is easy to check that $\rho$ is a metric. Hence, the proposition follows from the following two statements:

(1) any open ball $B\left(<_{0}, 1 / 2^{r}\right)$ (with respect to the metric $\rho$ ) is open in the topology introduced in Definition 1.1

Proof: $<_{1} \in B\left(<_{0}, 1 / 2^{r}\right)$ if and only if $<_{1}$ and $<_{0}$ coincide on the set $G_{r+1}$. Therefore

$$
B\left(<_{0}, 1 / 2^{r}\right)=\bigcap U_{a b},
$$

where the intersection is taken over all possible pairs $(a, b)$ of elements of $G_{r+1}$ for which $a<_{0} b$.

(2) any set of the form $U_{a_{1} b_{1}} \cap U_{a_{2} b_{2}} \cap \ldots \cap U_{a_{n} b_{n}}$ is open with respect to the metric $\rho$. In other words, for any $<_{0} \in U_{a_{1} b_{1}} \cap U_{a_{2} b_{2}} \cap \ldots \cap U_{a_{n} b_{n}}$ there exist $r$ such that $B\left(<_{0}, 1 / 2^{r}\right) \subset U_{a_{1} b_{1}} \cap U_{a_{2} b_{2}} \cap \ldots \cap U_{a_{n} b_{n}}$.

Proof: There exists an element of the filtration, $G_{r}$, such that $a_{1}, b_{1}, \ldots, a_{n}, b_{n} \in G_{r}$. For such $r, B\left(<_{0}, 1 / 2^{r}\right) \subset U_{a_{1} b_{1}} \cap U_{a_{2} b_{2}} \cap \ldots \cap U_{a_{n} b_{n}}$.

Recall that a space is totally disconnected if every two distinct points of it are contained in two disjoint open sets covering the space.

Theorem 1.4. $L O(G)$ is a compact, totally disconnected topological space.

Proof: For any two left orderings $<_{1},<_{2} \in L O(G)$ there exist $a, b \in G$ such that $<_{1} \in U_{a b}$ and $<_{2} \in U_{b a}$. Since $U_{a b} \cup U_{b a}=L O(G), U_{a b} \cap U_{b a}=\emptyset, L O(G)$ is totally disconnected. Now we are going to show that $L O(G)$ is compact. Consider any complete, infinite filtration of $G$ by its finite subsets and the associated metric $\rho$. We need to prove that any infinite sequence $<_{1},<_{2}$ ,$\ldots \in L O(G)$ has a convergent subsequence. We construct this subsequence in the following manner: Since there are only finitely many possible linear orderings of elements of $G_{1}$ there is an infinite subsequence $<_{i_{1}^{1}},<_{i_{2}^{1}},<_{i_{3}^{1}} \ldots$ of $<_{1},<_{2}, \ldots$ whose elements induce the same linear order on $G_{1}$. Now, pick out of this sequence an infinite subsequence of orders, $<_{i_{1}^{2}},<_{i_{2}^{2}}, \ldots$, which agree on $G_{2}$. Continue this process for $G_{3}, G_{4}, \ldots$ ad infinitum. Consider now a sequence $<^{1},<^{2},<^{3}, \ldots$ constructed by picking up the $n$-th element from the $n$-th subsequence constructed above for $n=1,2, \ldots$ Since $<^{1},<^{2},<^{3}, \ldots$. is a subsequence of $<_{1},<_{2}, \ldots$ the following lemma completes the proof.

Lemma 1.5. $<^{1},<^{2},<^{3}, \ldots$ converges to a left order $<^{\infty}$, defined as follows: $a<^{\infty} b$ if and only if $a<^{n} b$ for almost all $n$.

Proof. If $a, b \in G_{r}$ then either $a<^{i} b$ for $i>r$ or $b<^{i} a$ for $i>r$. Therefore, $<^{\infty}$ is a total order and, it is easy to verify that it is also a left order on $G$. Since $\rho\left(<^{n},<^{\infty}\right) \leq \frac{1}{2^{n}}$, the sequence $<^{1},<^{2},<^{3}, \ldots$ converges to $<^{\infty}$.

Corollary 1.6. $L O(G)$ is homeomorphic to the Cantor set if and only if (1) $L O(G) \neq \emptyset$, and

(2) for any sequence $a_{1}, b_{1}, \ldots ., a_{n}, b_{n}$, of elements of $G$, the set $U_{a_{1} b_{1}} \cap U_{a_{2} b_{2}} \cap$ $\ldots \cap U_{a_{n} b_{n}}$ is either empty or infinite. 
Proof. Any nonempty, metrizable, compact, perfect and totally disconnected set is a Cantor set (6, Corollary 2-98]). (A set is perfect if every point of the set is a limit point.) Condition (2) states that $L O(G)$ is perfect.

Orderings on $\mathbb{Z}^{n}$ were classified in [14, 10].

Proposition 1.7. For $n>1, L O\left(\mathbb{Z}^{n}\right)$ is homeomorphic to the Cantor set.

Proof. Consider the smallest $n>1$ for which the statement fails. By Corollary [1.6] there exists a finite set of pairs $\left(a_{1}, b_{1}\right), \ldots,\left(a_{s}, b_{s}\right) \in \mathbb{Z}^{n} \times \mathbb{Z}^{n}$ such that the number of orderings $<$ on $\mathbb{Z}^{n}$ such that $a_{i}<b_{i}$, for $i=1, \ldots, s$, is positive and finite. By adding some additional pairs, if necessary, we can assume that there is only one such ordering, $<$. Furthermore, we can assume that $b_{j}-a_{j}$ is not a rational multiple of $b_{i}-a_{i}$, for any $i \neq j$. We extend $<$ to an ordering on $\mathbb{Q}^{n}$ by demanding that $v_{1}<v_{2}$ for $v_{1}, v_{2} \in \mathbb{Q}^{n}$ if and only if $n v_{1}<n v_{2}$ for all $n \in \mathbb{Z}$ such that $n v_{1}, n v_{2} \in \mathbb{Z}^{n}$. Consider the set $H \subset \mathbb{Q}^{n} \otimes \mathbb{R}=\mathbb{R}^{n}$ composed of elements $x$ such that any neighborhood of $x$ in $\mathbb{Q}^{n}$ (with respect to the Euclidean topology in $\mathbb{R}^{n}$ ) contains both positive and negative elements. $\left(v \in \mathbb{Q}^{n}\right.$ is positive if $\left.0<v\right)$. One can prove that $H$ is a hyper-plane in $\mathbb{R}^{n}$ and that the two connected components of $\mathbb{R}^{n} \backslash H$, denoted by $H_{+}$and $H_{-}$, have the property that all elements of $\mathbb{Q}^{n}$ in $H_{+}$are positive and all elements of $\mathbb{Q}^{n}$ in $H_{-}$are negative. Therefore the vectors $b_{i}-a_{i}$ lie either in $H_{+}$or in $H$. Denote by $I$ the set of $i$ 's such that $b_{i}-a_{i} \in H$. Observe that $<$ is the only order on $H \cap \mathbb{Z}^{n}$ for which $a_{i}<b_{i}$ for $i \in I$. (Any other order on $H \cap \mathbb{Z}^{n}$ satisfying this condition would extended to an order $<^{\prime}$ on $\mathbb{Q}^{n}$ for which $a_{i}<^{\prime} b_{i}$ for all $i=1, \ldots, k$. This would contradict the uniqueness of $<$ ). Since $H \cap \mathbb{Z}^{n}$ does not satisfy condition (2) of Corollary [1.6] the initial assumption about $n$ implies that $H \cap \mathbb{Z}^{n}=\emptyset$ or $\mathbb{Z}$. If $H \cap \mathbb{Z}^{n}=\emptyset$ then $I=\emptyset$ and there exist infinitely many hyper-planes $H^{\prime} \subset \mathbb{R}^{n}$, obtained by small perturbations of $H$, giving rise to infinitely many orderings $<^{\prime}$ of $\mathbb{Q}^{n}$ such that $a_{i}<^{\prime} b_{i}$, for $i=1, \ldots, k$. Therefore, $H \cap \mathbb{Z}^{n}=\mathbb{Z}$. Since we assumed that none of the vectors $b_{i}-a_{i}$ is a rational multiple of another, we see that $b_{i}-a_{i}$ lies in $H$ for exactly one index $i=i_{0}$. Now, by small perturbations of $H$ in $\mathbb{R}^{n}$ we can obtain infinitely many new hyper-planes $H^{\prime}$ such that $b_{i_{0}}-a_{i_{0}}$ lies in the same component of $\mathbb{R}^{n} \backslash H^{\prime}$ as all other vectors $b_{i}-a_{i}$. Each of these hyper-planes induces an ordering $<^{\prime}$ on $\mathbb{Z}^{n}$ such that $a_{i}<^{\prime} b_{i}$ for $i=1, \ldots, k$ - a contradiction.

\section{BI-ORDERINGS}

A bi-ordering is a linear ordering which is both left and right ordering. Using Proposition [1.3 we see that the set of bi-orderings $\mathrm{BiO}(G)$ inherits the same topology from $L O(G)$ and $R O(G)$.

Proposition 2.1. $\mathrm{BiO}(G)$ is a closed subset of $L O(G)$. Hence $\mathrm{BiO}(G)$ is the Cantor set if and only if $\mathrm{BiO}(G) \neq \emptyset$ and for any sequence $a_{1}, b_{1}, \ldots, a_{n}, b_{n}$, of elements of $G$, the set $\mathrm{BiO}(G) \cap U_{a_{1} b_{1}} \cap U_{a_{2} b_{2}} \cap \ldots \cap U_{a_{n} b_{n}}$ is either empty or infinite. 
Proof. Consider a metric on $L O(G)$ induced by a filtration of $G$. For any infinite sequence, $<_{1},<_{2}, \ldots$, of bi-orders of $G$ converging to a left order, $<_{\infty}$, we have $a<_{\infty} b$ if and only if $a<_{i} b$ for almost all $i$. Hence $a<_{\infty} b$ implies $a c<_{\infty} b c$ for any $c$. This proves that $\mathrm{BiO}(G)$ is a closed subset of $L O(G)$. The second part of the statement is proved exactly as Corollary [1.6.

One should not expect, however, that the set of bi-orderings of a group, if infinite, is homeomorphic to the Cantor set. There are known examples of groups $G$ for which $\mathrm{BiO}(G)$ has countably infinite number of elements, [8.

Conjecture 2.2. For the free group on $n>1$ generators, $F_{n}$, the spaces $L O\left(F_{n}\right)$ and $\mathrm{BiO}\left(F_{n}\right)$ are homeomorphic to the Cantor set.

Let $G=G^{0} \supset G^{1} \supset \ldots$ be the lower central series of a group $G$ such that $\bigcap_{k=0}^{\infty} G^{k}=1$. Šimbireva and Neumann (see [13, 9] and [4, Ch. IV §2]) showed that any choice of orders on the groups $G^{k} / G^{k+1}$ yields a total biorder on $G$ : if $g \in G^{k} \backslash G^{k+1}$ then $g$ is positive in $G$ if and only if $g$ is positive in $G^{k} / G^{k+1}$. Since any torsion-free abelian group is orderable, 14, if the groups $G^{k} / G^{k+1}$ are torsion-free then $G$ is bi-orderable ${ }^{1}$. We will call such orders standard and denote their set by $\operatorname{SBiO}(G)$. They are characterized by the following condition satisfied by all $g \in G$ : if $g \in G^{k} \backslash G^{k+1}$ and $g$ is positive then all elements of $g G^{k+1}$ are positive.

Proposition 2.3. (1) $\mathrm{SBiO}(G)$ is a closed subset of $\mathrm{BiO}(G)$.

(2) If $G \neq \mathbb{Z}$ and each factor $G^{k} / G^{k+1}$ is finitely generated then $\operatorname{SBiO}(G)$ is either empty or homeomorphic to the Cantor set.

It is possible that the results of 14 make possible to relax the assumption about the finite number of generators of each of the factors $G^{k} / G^{k+1}$. Proof: (1) If an order $\leq$ is not standard then, by the above characterization of standard orders, there exists $g \in G^{k} \backslash G^{k+1}$ and $h \in G^{k+1}$ such that $g>e$ and $g h<e$. Therefore $\leq$ has an open neighborhood composed of non-standard orders.

(2) Assume that $G \neq \mathbb{Z}$. We may also assume that all $G^{k} / G^{k+1}$ are torsion-free and, hence, free abelian groups, since otherwise $\operatorname{SBiO}(G)=\emptyset$. As before, it suffices to prove that for any $a_{1}, b_{1}, \ldots, a_{n}, b_{n} \in G$ the set

$$
\operatorname{SBiO}(G) \cap U_{a_{1} b_{1}} \cap U_{a_{2} b_{2}} \cap \ldots \cap U_{a_{n} b_{n}}
$$

is either empty or infinite. Assume that this set is not empty. If $G^{k} / G^{k+1}=$ $\mathbb{Z}^{n}$ for $n \geq 2$ for some $k$ then one can obtain infinitely many standard orders in $U_{a_{1} b_{1}} \cap U_{a_{2} b_{2}} \cap \ldots \cap U_{a_{n} b_{n}}$ by slightly modifying the order on $G^{k} / G^{k+1}$. (We use here the classification of orders of $\mathbb{Z}^{n}$ given in the proof of Proposition 1.7). Therefore from now on it is enough to assume that $G^{k} / G^{k+1}$ is either $\mathbb{Z}$ or trivial for all $k$.

\footnotetext{
${ }^{1}$ In fact the result of Šimbireva and Neumann applies to all groups with a central series, including the transfinite ones, ending with the trivial group.
} 
We claim that in this situation $G^{k} / G^{k+1}=\mathbb{Z}$ for all $k$. Indeed if $G^{k} / G^{k+1}$ is trivial for some $k$ then $G^{l}=G^{k}$ for all $l \geq k$, and since $\bigcap_{i=0}^{\infty} G^{i}=1, G$ is nilpotent. However, the only nilpotent group $G$ with $G / G^{1}=\mathbb{Z}$ is $G=\mathbb{Z}$. (This statement follows from [5. Cor. 10.3.3] where $H=<h>$ and $h G^{1}$ is a generator of $G / G^{1}=\mathbb{Z}$ ).

Therefore, we may assume that $G^{k} / G^{k+1}=\mathbb{Z}$ for all $k$. Since each $G^{k} / G^{k+1}=\mathbb{Z}$ has exactly two orderings it is not hard to see that $\operatorname{SBiO}(G)=$ $\{0,1\}^{\aleph_{0}}$ as a topological space.

An example of a non-standard order can be constructed as follows: Vinogradov proved that if $A, B$ are bi-ordered groups then $A * B$ has also a bi-order, [15, 8. The construction of such bi-order given in the proof of Theorem 2.3.1 in [8] has the property that if $a_{0}, a_{1} \in A, b \in B, a_{0}>e$, $a_{1}<e, b>e$ then $a_{0} b a_{1} b^{-1} a_{1}^{-1}<e$. Therefore that order is non-standard.

\section{EXAMPLE: $\mathbb{Z} \times \mathbb{Z}$}

Let $\mathbb{R}_{[\text {) }}$ denote the real line with the topology with a basis of open sets composed of intervals $[a, b)$. Analogously, let $\mathbb{R}_{(]}$be the real line with the topology whose basis is composed of intervals $(a, b]$. The above topologies carry onto the unit circle in $\mathbb{R}^{2}$ via the map $z \rightarrow e^{2 \pi i z}, \mathbb{R}_{[)} / \mathbb{Z} \rightarrow S_{[)}^{1} \subset \mathbb{R}^{2}$, $\mathbb{R}_{(]} / \mathbb{Z} \rightarrow S_{(]}^{1} \subset \mathbb{R}^{2}$. We say that a point $(x, y) \in S^{1} \subset \mathbb{R}^{2}$ is rational if $x / y \in \mathbb{Q}$.

Let $X$ be the union of these two circles with corresponding irrational points identified, $X=\left(S_{[)}^{1} \sqcup S_{(]}^{1}\right) / \sim$, where $\left(x_{1}, y_{1}\right) \sim\left(x_{2}, y_{2}\right)$ if $\left(x_{1}, y_{1}\right) \in S_{[)}^{1}$, $\left(x_{2}, y_{2}\right) \in S_{(]}^{1}$, and $\left(x_{1}, y_{1}\right)=\left(x_{2}, y_{2}\right)$ is irrational.

Proposition 3.1. $\mathrm{BiO}\left(\mathbb{Z}^{2}\right)$ is homeomorphic to $X$.

Proof. First, we construct a map $S_{[)}^{1} \sqcup S_{(]}^{1} \rightarrow B i O\left(\mathbb{Z}^{2}\right)$ as follows: Associate with $x \in S_{[\text {[) }}^{1}$ an order $<_{x}$ on $\mathbb{Z}^{2}$ such that $y \in \mathbb{Z}^{2}$ is positive if and only if the (oriented) angle between the vectors $y$ and $x$ in $\mathbb{R}^{2}$ is in the interval $(0, \pi]$. Similarly, we associate with $x \in S_{(]}^{1}$ an order $<^{x}$ on $\mathbb{Z}^{2}$ such that $y \in \mathbb{Z}^{2}$ is positive if and only if the angle between $y$ and $x$ is in the interval $[0, \pi)$. Note that these maps descend to a 1-1 map $i$ from $X$ into $\mathrm{BiO}(G)$.

We claim that $i$ is onto: Recall from the proof of Proposition [1.7 that any ordering on $\mathbb{Z}^{2}$ defines a 1-dimensional subspace $H$ in $\mathbb{R}^{2}$, such that one component of $\mathbb{R}^{2} \backslash H$ is composed of positive elements and the other of negative elements. Notice that all orderings on $\mathbb{Z}^{2}$ inducing the line $H$ are of the form $<_{x}$ and $<^{x}$, where $x$ is one of the two unit vectors in $H$.

Finally, we claim that $i$ is a continuous map. To verify that claim it is sufficient to check that $i^{-1}\left(U_{0 a}\right) \subset X$ is open for any $a \in \mathbb{Z}^{2}$. This condition follows from the fact that the sets $\left\{x \in S_{[)}^{1}: 0<_{x} a\right\}$ and $\left\{x \in S_{(]}^{1}: 0<^{x} a\right\}$ are open in $S_{[)}^{1}$ and in $S_{(]}^{1}$, respectively. 
The above proposition has an unexpected application. Although it is known that any compact set is a continuous image of a map defined on the Cantor set (see [6. Thm 3-28]), usually it is difficult to visualize such a map. However, we can visualize a map from the Cantor set onto $S^{1}$. Consider the map $f: X \rightarrow S^{1}$ given by identification of all corresponding points, $x \in S_{[)}^{1}$ with $x \in S_{(]}^{1}$, in $X=S_{[)}^{1} \sqcup S_{(]}^{1} / \sim$. Since the topology of $S_{(]}^{1}$ and of $S_{[)}^{1}$ is richer than the Euclidean topology of the circle, $f$ is continuous. By Propositions 1.7 and $3.1 X$ is the Cantor set.

\section{Applichtions to Gröbner bases}

Let $\mathbb{K}\left[x_{1}, \ldots, x_{n}\right]$ be a polynomial ring over a field $\mathbb{K}$. Monomials in it, $x_{1}^{i_{1}} \ldots x_{n}^{i_{n}}$, form a monoid (a semi-group with an identity), isomorphic to $\mathbb{Z}_{\geq 0}^{n}$ by the isomorphism which carries $x_{1}^{i_{1}} \ldots x_{n}^{i_{n}}$ to $\left(i_{1}, \ldots, i_{n}\right)$. From now on we will identify these two monoids.

A linear order on a set $G$ is a well-order if any subset of $G$ has a smallest element. For a semi-group $G$, we denote the set of all left well-orders of $G$ by $L W O(G)$. The elements of $L W O\left(\mathbb{Z}_{\geq 0}^{n}\right)$ are called monomial orderings on $\mathbb{K}\left[x_{1}, \ldots, x_{n}\right]$. As a consequence of Dickson's lemma, 3, Cor. 6 Ch.2 $\S 4$ ], an order $<$ on $\mathbb{Z}_{\geq 0}^{n}$ is a well-order if and only if 0 is the smallest element for $<$. Therefore

$$
L W O\left(\mathbb{Z}_{\geq 0}^{n}\right)=L O\left(\mathbb{Z}_{\geq 0}^{n}\right) \backslash \bigcup U_{a 0}
$$

where the sum is over all non-zero elements of $\mathbb{Z}_{\geq 0}^{n}$. Since $\bigcup U_{a 0}$ is open, we get the following.

Corollary 4.1. $L W O\left(\mathbb{Z}_{\geq 0}^{n}\right)$ is a closed subset of $L O\left(\mathbb{Z}_{\geq 0}^{n}\right)$. Hence, by Theorem 1.4, $L W O\left(\mathbb{Z}_{\geq 0}^{n}\right)$ is compact.

Furthermore, by adopting the reasoning given in the proof of Proposition 1.7. one can show that both $L W O\left(\mathbb{Z}_{\geq 0}^{n}\right)$ and $L O\left(\mathbb{Z}_{\geq 0}^{n}\right)$ are homeomorphic to the Cantor set for $n>1$.

Any polynomial $w \in \mathbb{K}\left[x_{1}, \ldots, x_{n}\right]$ decomposes as $\sum_{1}^{d} c_{i} m_{i}$, where $m_{i}$ 's are monomials, $m_{i} \neq m_{j}$, and $c_{i}$ 's are scalars in $\mathbb{K}, c_{i} \neq 0$. For a given monomial ordering, the leading monomial of $w, L M(w)$, is the largest monomial among $m_{1}, \ldots, m_{d}$. If $L M(w)=m_{i}$, then the leading term of $w$ is $L T(w)=c_{i} m_{i}$. For a non-zero ideal $I \triangleleft \mathbb{K}\left[x_{1}, \ldots, x_{n}\right]$, we denote by $L M(I)$ the ideal in $\mathbb{K}\left[x_{1}, \ldots, x_{n}\right]$ generated by the leading monomials of polynomials in $I$. Finally, we say that a set of polynomials in $\left\{f_{1}, \ldots, f_{d}\right\} \subset I$, is a Gröbner basis of $I$ if the leading monomials of these polynomials generate $L M(I)$. Such a basis is very useful for practical computations with $I$ and $\mathbb{K}\left[x_{1}, \ldots, x_{n}\right] / I$, see [1, 2, 3]. Buchberger's algorithm provides a practical method of calculating a Gröbner bases, see eg. [3, Ch. $2 \oint 7]$.

Proposition 4.2. For any ideal $I \triangleleft \mathbb{K}\left[x_{1}, \ldots, x_{n}\right]$ and for any set of polynomials $f_{1}, \ldots, f_{s} \in I$ the set of monomial orderings on $\mathbb{K}\left[x_{1}, \ldots, x_{n}\right]$ for which $f_{1}, \ldots, f_{s}$ is a Gröbner basis of $I$ is open. 
Proof. For any $f, g \in \mathbb{K}\left[x_{1}, \ldots, x_{n}\right]$, let

$$
S(f, g)=\frac{L C M(L M(f), L M(g))}{L T(f)} f-\frac{L C M(L M(f), L M(g))}{L T(g)} g,
$$

where $L C M$ denotes the least common multiple. By [3, Theorem 2.§6.6], a set $\left\{f_{1}, \ldots, f_{s}\right\}$ is a Gröbner basis for $I$ if and only if $S\left(f_{i}, f_{j}\right)$ is divisible by $\left\{f_{1}, \ldots, f_{s}\right\}$ (with remainder $=0$ ). (The division by multiple polynomials was defined in [3, Theorem 2.§3.3]). The division process is based on a finite number of comparisons $m \leq m^{\prime}$ between monomials. Therefore, if $S\left(f_{i}, f_{j}\right)$ is divisible by $\left\{f_{1}, \ldots, f_{s}\right\}$ for a monomial ordering $\leq$, then it is also divisible by $\left\{f_{1}, \ldots, f_{s}\right\}$ for all orderings in some open neighborhood of $\leq$ in the space of monomial orderings.

As an application of Theorem 1.4 we can give now a very short proof of the existence of universal Gröbner bases (following the idea of the proof given in [12]).

Theorem 4.3. 12] For any ideal $I \triangleleft \mathbb{K}\left[x_{1}, \ldots, x_{n}\right]$ there exists a finite set of polynomials $f_{1}, \ldots, f_{s} \in I$ which is a Gröbner basis of $I$ with respect to any monomial ordering. Such set is called a universal Gröbner basis.

Proof. For any $f_{1}, \ldots, f_{s} \in I$ let $V_{\left\{f_{1}, \ldots, f_{s}\right\}}$ be the set of monomial orderings for which $\left\{f_{1}, \ldots, f_{s}\right\}$ is a Gröbner basis of $I$. By Proposition 4.2, $V_{\left\{f_{1}, \ldots, f_{s}\right\}}$ is open. Since, by Corollary 4.1, the space of monomial orderings is compact, it has a finite cover by $V_{\left\{f_{1}, \ldots, f_{s}\right\}}, \ldots, V_{\left\{h_{1}, \ldots, h_{t}\right\}}$. The set $\left\{f_{1}, \ldots, f_{s}, \ldots, h_{1}, \ldots, h_{t}\right\}$ is the universal Gröbner basis of $I$.

The following example shows that Corollary 4.1 (which was an essential part of the above proof) does not hold for some monoids other then $\mathbb{Z}_{>0}^{n}$.

Example: The monoid of monomials in $\mathbb{K}\left[x_{1}, x_{2}, \ldots ..\right]$ is isomorphic to the infinite sum, $\mathbb{Z}_{\geq 0}^{\infty}$. Each linear order, $<$, on the set $\left\{x_{1}, x_{2}, \ldots\right\}$ induces a lexicographic order on $\mathbb{K}\left[x_{1}, x_{2}, \ldots.\right]$ : Let $m=x_{1}^{m_{1}} x_{2}^{m_{2}} \ldots$ and $n=x_{1}^{n_{1}} x_{2}^{n_{2}} \ldots$ be two arbitrary monomials in $\mathbb{K}\left[x_{1}, x_{2}, \ldots.\right]$. Let $x_{i}$ be the smallest variable (with respect to $<$ ) for which $m_{i} \neq n_{i}$. Then $m<n$ if and only if $m_{i}<n_{i}$. We denote such induced order on $\mathbb{K}\left[x_{1}, x_{2}, \ldots.\right]$ by $<_{L}$ (L stands for lexicographic). $<_{L}$ is a well-order if and only if $<$ is a well-order. Consider now a sequence of well-orders $<^{1},<^{2}, \ldots$ such that

$$
x_{n}<{ }^{n} x_{n-1}<{ }^{n} \ldots<^{n} x_{2}<{ }^{n} x_{1}<{ }^{n} x_{n+1}<{ }^{n} x_{n+2}<{ }^{n} x_{n+3}<{ }^{n} \ldots
$$

Consider a filtration $\left\{G_{*}\right\}$ of $\mathbb{Z}_{\geq 0}^{\infty}$ such that $G_{n}$ contains all monomials in variables $x_{1}, \ldots, x_{n}$ of total degree at most $n$. It is easy to check that the sequence of lexicographic orders $<_{L}^{1},<_{L}^{2}, \ldots$ converges with respect to the metric induced by the filtration $\left\{G_{*}\right\}$ to a new lexicographic order $<_{L}^{\infty}$ induced by

$$
\ldots<^{\infty} x_{n}<^{\infty} x_{n-1}<^{\infty} \ldots<^{\infty} x_{2}<^{\infty} x_{1} .
$$

Hence $<_{L}^{\infty}$ is not a well-order on $\mathbb{K}\left[x_{1}, x_{2}, \ldots.\right]$, and therefore, $L W O\left(\mathbb{Z}_{\geq 0}^{\infty}\right)$ is not a closed subset of $L O\left(\mathbb{Z}_{\geq 0}^{\infty}\right)$. 


\section{Acknowledgments}

We thank R. Bryant for suggesting a simplification of the proof of Proposition 2.3 (2) and the referee of this paper for many insightful comments.

\section{REFERENCES}

[1] W. Adams, P. Loustaunau, An Introduction to Gröbner bases, Graduate Studies in mathematics 3, AMS, 1994.

[2] T. Becker, V. Weispfenning, Gröbner Bases, A Computational Approach to Algebra, Graduate Texts in Mathematics, Springer-Verlag 1993.

[3] D. Cox, J. Little, D. O'Shea, Ideals, Varieties, and Algorithms, An Introduction to Computational Algebraic Geometry and Commutative Algebra, 2nd edition, Springer 1997.

[4] L. Fuchs, Partially Ordered Algebraic Systems, Pergamon Press 1963.

[5] M. Hall Jr., The theory of groups, Macmillan Co. 1959.

[6] J. G. Hocking, G. S. Young, Topology, Addison-Wesley Publishing Company Inc., 1961.

[7] A. I. Kokorin, V. M. Kopytov, Fully ordered groups, Halsted Press (John Wiley \& Sons), New York-Toronto, Ont.; Israel Program for Scientific Translations, JerusalemLondon, 1974.

[8] R. B. Mura, A. Rhemtulla, Orderable groups, Lectures in pure and applied mathematics, Vol. 27, Marcel Dekker Inc., 1977.

[9] B. H. Neumann, On ordered groups, Amer. Journ. Math. 71(1949) 202-252.

[10] L. Robbiano, Term orderings on the polynomial ring. In: B. F. Cavines ed., Eurocal'85, European Conference on Computer Algebra, Vol. II, Springer LNCS 204, $513-517$.

[11] D. J. S. Robinson, A course in the theory of groups, Second Ed., Graduate Texts in Mathematics, Springer 1996.

[12] N. Schwartz, Stability of Gröbner bases, J. of Pure and Applied Alg. 53(1988), 171186.

[13] E.P. Šimbireva, On the theory of partially ordered groups. (In Russian), Mat. Sbornik 20(1947), 145-178.

[14] H.-H. Teh, Construction of orders in abelian groups, Proc. Camb. Phil. Soc. 57(1961), 467-482.

[15] A. A. Vinogradov, On the free product of ordered groups, Mat. Sbornik 67 (N.S. 25) 1949, 163-168.

Dept. of Mathematics, 244 Math Bldg, SUNY Buffalo, NY 14260 asikora@buffalo.edu 